\title{
EQUILIBRIUM STATES OF AN ELASTIC CONDUCTOR IN A MAGNETIC FIELD: A PARADIGM OF BIFURCATION THEORY
}

BY

PETER WOLFE

\begin{abstract}
In this paper we study the equilibrium states of a nonlinearly elastic conducting wire in a magnetic field. The wire is perfectly flexible and is suspended between fixed supports. The wire carries an electric current and is subjected to a constant magnetic field whose direction is parallel to the line between the supports. We solve this problem exactly and show that the set of solutions gives rise to a paradigmatic bifurcation diagram. We then carry out a study of the equations obtained by linearization about the nontrivial solutions in order to gain some insight into the stability of the various solution branches.
\end{abstract}

Introduction. In this paper we study the equilibrium states of a nonlinearly elastic conducting wire in a magnetic field. The wire is assumed to be perfectly flexible and is suspended between fixed supports. The distance between the supports is assumed to be greater than the natural length of the wire and therefore the wire will always be in tension. The magnetic field is assumed to be constant and is directed parallel to the line between the supports. We show that this problem can be solved exactly. The set of solutions exhibits the classic bifurcation phenomenon. The bifurcation parameter is $\lambda=I B$ where $I$ is the current in the wire and $B$ the strength of the magnetic field. For all values of $\lambda>0$ there is a trivial solution in which the wire remains straight. However at the eigenvalues of the problem obtained by linearizing the equilibrium equation about the trivial solution, bifurcation occurs and we obtain branches of nontrivial solutions. The situation is quite similar to the classical problem of the buckling of a beam. Actually in our case the branches of solutions are two-dimensional manifolds because the problem is invariant under rotation about the axis of the supports.

This work was motivated by the discussion in [1] (see \$4.7, especially Figure 29 and the reference quoted therein).

There are two cases to consider as the wire is (elastically) homogeneous or not. Of course it is of interest to study the stability of the equilibria with respect to the (hyperbolic) equations of motion. This is in general a very difficult problem and in the present case it is complicated even further by the multiplicity of equilibria arising from the rotational invariance. In the case of the homogeneous wire we can study the operator obtained by linearization about a nontrivial solution. We are able to locate 
the spectrum of such an operator. This represents a first step in the stability analysis and leads to a conjecture about the stability of the branches.

In $\S 1$ we formulate and solve the governing equations while $\$ 2$ is devoted to the linearization of the equations and a discussion of stability.

1. Formulation and solution of the governing equations. Let a nonlinearly elastic conducting wire have a natural length 1 . We identify each material point of the wire by its coordinate $s \in[0,1]$. Let $\mathbf{r}(s)$ represent the deformed position in Euclidean 3-space $\mathbf{E}^{3}$ of the material point $s$. Let $\nu(s)=\left|\mathbf{r}^{\prime}(s)\right|\left(^{\prime}=d / d s\right)$ and $\mathbf{e}(s)=$ $\mathbf{r}^{\prime}(s) / \nu(s)$. The wire is assumed stretched between fixed supports located at $\mathbf{0}$ and $b \mathbf{k}$ where $b \geqslant 1$. (Here $\{\mathbf{i}, \mathbf{j}, \mathbf{k}\}$ represents the standard orthonormal basis for $E^{3}$.) The wire carries a current $I$. There is a constant magnetic field $\mathbf{B}=B \mathbf{k}$ present. The force on the wire is then given by

$$
\mathbf{f}(s)=I \mathbf{r}^{\prime} \times B \mathbf{k}=I \nu \mathbf{e} \times B \mathbf{k} .
$$

Let $\mathbf{n}(s)$ denote the resultant contact force exerted by the material of $(s, 1]$ on the material of $[0, s]$. Then the equation of equilibrium for the wire has the form

$$
\mathbf{n}^{\prime}(s)+\mathbf{f}(s)=\mathbf{0} \text {. }
$$

We assume the wire is perfectly flexible. This means that it has the property that

$$
\mathbf{n}(s)=N(s) \mathbf{e}(s) \text {. }
$$

Recall that $\mathbf{e}(s)$ is the unit tangent to the wire at $s$. The function $N(s)$ is the tension at $s$. We assume that the wire is nonlinearly elastic so that the tension at $s$ depends on the stretch $\nu(s)$. Specifically we assume that there is a continuously differentiable function $(0, \infty) \times[0,1] \ni(\nu, s) \rightarrow \hat{N}(\nu, s) \in R$ with

$$
\begin{aligned}
\hat{N}_{\nu}(\nu, s)>0, & \hat{N}(1, s)=0, \\
\hat{N}(\nu, s) \rightarrow \infty \quad \text { as } \nu \rightarrow \infty, & \hat{N}(\nu, s) \rightarrow-\infty \quad \text { as } \nu \rightarrow 0
\end{aligned}
$$

so that

$$
N(s)=\hat{N}(\nu(s), s) .
$$

Our boundary value problem is thus to find functions $\mathbf{r}(s)$ satisfying

$$
\begin{array}{cc}
(N(s) \mathbf{e})^{\prime}+I \nu \mathbf{e} \times B \mathbf{k}=\mathbf{0}, & 0<s<1, \\
\mathbf{r}(0)=\mathbf{0}, \quad \mathbf{r}(1)=b \mathbf{k}, & b>1 .
\end{array}
$$

We seek classical solutions of (1.7), (1.8). Let us first consider the case of the homogeneous wire, i.e., we assume the function $\hat{N}$ is independent of $s$. We rewrite (1.7) in the form

$$
N^{\prime}(s) \mathbf{e}+N(s) \mathbf{e}^{\prime}+\lambda \nu(\mathbf{e} \times \mathbf{k})=\mathbf{0}
$$

where

$$
\lambda=I B>0 .
$$

We take the dot product of (1.9) with e. This gives

$$
N^{\prime}(s)=0 \text {. }
$$

Thus $N$ is a constant. Since $\hat{N}$ is independent of $s,(1.4),(1.6)$ imply that $\nu$ is a constant. Since $b>1$ we must have $\nu \geqslant b>1$ so that $N>0$. Now take the dot 
product of (1.9) with $\mathbf{k}$ and use (1.11). This gives

$$
N \mathbf{e}^{\prime} \cdot \mathbf{k}=0
$$

so that

$$
\mathbf{e} \cdot \mathbf{k}=\text { const } \equiv \cos \psi .
$$

If $\cos \psi=1$ then $\mathbf{e}=\mathbf{k}$ and we have the "trivial" solution (with $\nu \equiv b$ )

$$
\mathbf{r}(s)=b s \mathbf{k} \text {. }
$$

The solution (1.14) exists for all values of $\lambda$. Now suppose that $|\cos \psi|<1$. We can then write

$$
\mathbf{e}=\cos \theta \sin \psi \mathbf{i}+\sin \theta \sin \psi \mathbf{j}+\cos \psi \mathbf{k} .
$$

Using (1.15) in (1.9) we obtain

(1.16) $N\left[-\theta^{\prime} \sin \theta \sin \psi \mathbf{i}+\theta^{\prime} \cos \theta \sin \psi \mathbf{j}\right]+\lambda \nu[\sin \theta \sin \psi \mathbf{i}-\cos \theta \sin \psi \mathbf{j}]=\mathbf{0}$.

Taking the dot product of (1.16) with $\cos \theta \mathbf{j}-\sin \theta \mathbf{i}$ then yields

$$
\theta^{\prime}=\nu \lambda / N \text {. }
$$

Now from (1.8),

$$
\nu \int_{0}^{1} \mathbf{e}(s) d s=b \mathbf{k},
$$

from which it follows that, by taking components

(1.19a, b, c) $\quad \int_{0}^{1} \cos \theta d s=0, \quad \int_{0}^{1} \sin \theta d s=0, \quad \nu \cos \psi=b$.

Using (1.17) in (1.19a) and (1.19b) we see

$$
\begin{gathered}
\sin \left(\theta_{0}+\nu \lambda / N\right)-\sin \theta_{0}=0, \quad \theta_{0}=\theta(0), \\
\cos \left(\theta_{0}+\nu \lambda / N\right)-\cos \theta_{0}=0 .
\end{gathered}
$$

(1.20) and (1.21) can be written

$$
\left(\begin{array}{ll}
\sin \theta_{0} & \cos \theta_{0} \\
\cos \theta_{0} & -\sin \theta_{0}
\end{array}\right)\left(\begin{array}{l}
\cos (\nu \lambda / N)-1 \\
\sin (\nu \lambda / N)
\end{array}\right)=\left(\begin{array}{l}
0 \\
0
\end{array}\right) .
$$

From (1.22) we see that we must have (with $N=\hat{N}(\nu)$ )

$$
\nu \lambda / N=2 \pi m, \quad m=1,2,3, \ldots
$$

(1.19) then gives $\cos \psi$. We may then use (1.17) to integrate (1.15) to find r. Summing we have

1.24. ThEOREM. Suppose $N=\hat{N}(\nu)$. Let $\lambda>0$ and $\nu>b$ satisfy (1.23) for some positive integer $m$. Then the problem (1.7), (1.8) has solutions

$$
\begin{aligned}
\mathbf{r}_{\theta_{0}}(s)= & (N / \lambda)\left[\sin \left(\theta_{0}+\nu \lambda / N\right) s-\sin \theta_{0}\right] \sin \psi \mathbf{i} \\
& +(N / \lambda)\left[\cos \theta_{0}-\cos \left(\theta_{0}+\nu \lambda / N\right) s\right] \sin \psi \mathbf{j}+\nu \cos \psi s \mathbf{k}, \\
& 0 \leqslant \theta_{0}<2 \pi .
\end{aligned}
$$

Here

$$
\psi=\arccos b / \nu \text {. }
$$


Thus we see that for each pair $(\nu, \lambda)$ satisfying (1.23) for some $m$ we get a 1 -parameter family of solutions parameterized by $S^{1}$. The reason for this multiplicity is that the problem (1.7), (1.8) is invariant under rotations about the $z$ axis.

The set of solutions exhibits the classical bifurcation phenomenon. To see this we linearize (1.7) about the trivial solution (1.14) to obtain

$$
(\hat{N}(b) / b) x_{1}^{\prime \prime}+\lambda y_{1}^{\prime}=0, \quad(\hat{N}(b) / b) y_{1}^{\prime \prime}-\lambda x_{1}^{\prime}=0, \quad \hat{N}_{\nu}(b) z_{1}^{\prime \prime}=0,
$$

$x_{1}(0)=y_{1}(0)=z_{1}(0)=x_{1}(1)=y_{1}(1)=z_{1}(1)=0$. An elementary calculation shows that this problem has the eigenvalues

$$
\lambda_{m}=2 \pi m \hat{N}(b) / b
$$

with eigenfunctions

$$
\begin{aligned}
& x_{1}^{(m)}=c_{1}(1-\cos 2 \pi m s)+c_{2} \sin 2 \pi m s, \\
& y_{1}^{(m)}=-c_{1} \sin 2 \pi m s+c_{2}(1-\cos 2 \pi m s), \\
& z_{1}^{(m)}=0 .
\end{aligned}
$$

The values of $\lambda_{m}$ given by (1.28) are the bifurcation points. The branch of solutions on which (1.23) obtains bifurcates off from the trivial solution at $\lambda=\lambda_{m}$. For a given $\lambda$ the number of solution branches present depends on the behavior of the function $\hat{N}(\nu) / \nu$. If we assume

$$
\frac{d}{d \nu}(\hat{N}(\nu) / \nu)>0
$$

then we get the picture of Figure 1.31. For $0<\lambda \leqslant \lambda_{1}$ we have only the trivial solution. For $\lambda_{m}<\lambda \leqslant \lambda_{m+1}$ there are $m$ solution branches. Of course this picture is reminiscent of the buckling of a beam.

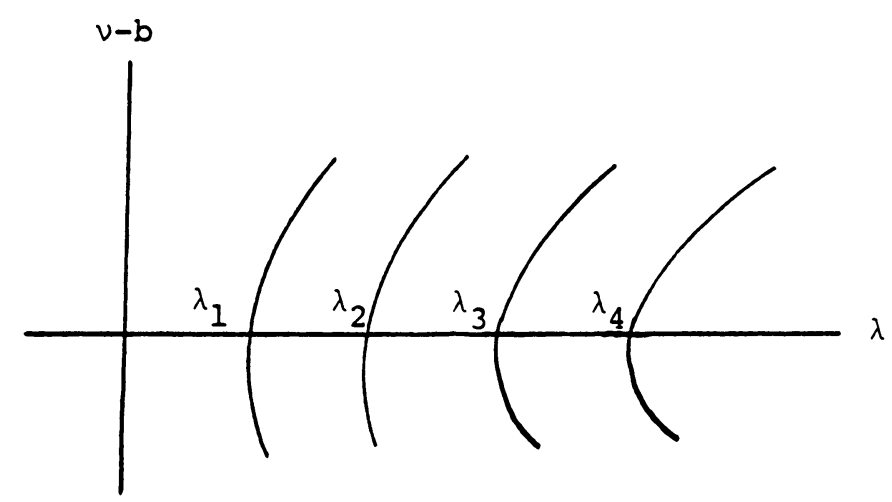

FIGURE 1.31

Bifurcation diagram if $d(\hat{N}(\nu) / \nu) / d \nu>0$. Note that each branch of nontrivial solution represents the one parameter family of solutions. To obtain a more accurate picture the figure should be rotated about the $\lambda$ axis. 
The problem (1.7), (1.8) admits the (rotationally invariant) potential energy functional

$$
\Phi=\int_{0}^{1}\left\{\Gamma(\nu, s)+\frac{\lambda}{2}(\mathbf{r} \times \mathbf{k}) \cdot \mathbf{r}^{\prime}\right\} d s
$$

with

$$
\Gamma(\nu, s)=\int_{1}^{\nu} \hat{N}(\sigma, s) d \sigma
$$

(1.7) is simply the Euler-Lagrange equation corresponding to the functional $\Phi$. It is a simple matter to compute $\Phi$ for the various equilibrium solutions. In fact from (1.25) we find that on the $m$ th branch (here we write $\Gamma=\Gamma(\nu)$ )

$$
\Phi=\Gamma(\nu)-(\lambda / 4 \pi m) \sin ^{2} \psi \nu^{2} .
$$

But using (1.23) and (1.26) this becomes

$$
\Phi=\Gamma(\nu)-\frac{1}{2} \nu \hat{N}(\nu)+\frac{1}{2} b^{2}(\hat{N}(\nu) / \nu) .
$$

Relation (1.35) also holds on the trivial branch where $\nu=b$. Recall (Figure 1.31) that for a given $\lambda \nu$ is largest on the branch with $m=1$. To show that $\Phi$ is minimal along this branch it is enough to show that $d F(\nu) / d \nu<0$ for $\nu>b$ where

$$
F(\nu)=\Gamma(\nu)-\frac{1}{2} \nu \hat{N}(\nu)+\frac{1}{2} b^{2}(\hat{N}(\nu) / \nu),
$$

but

$$
\frac{d}{d \nu} F(\nu)=\frac{1}{2}\left(b^{2}-\nu^{2}\right) \frac{d}{d \nu}(\hat{N}(\nu) / \nu)<0
$$

by (1.30). Thus we have

1.36. THEOREM. Assume (1.30) holds. Then for a given $\lambda>2 \pi \hat{N}(b) / b$ among all solutions of (1.7), (1.8) the potential energy $\Phi$ is minimized at solutions lying on the first solution branch (i.e., the set of solutions (1.25) with $\nu / N=2 \pi / \lambda)$.

Theorem 1.36 indicates that we may expect that the first branch of solutions is stable while the others are not. We will pursue this point further in $\$ 2$ when we consider the linearization of (1.7), (1.8) about nontrivial solutions.

We now turn to the general case in which the function $\hat{N}$ depends explicitly on $s$ as well as $\nu$. In this case (1.11) still holds but we cannot now conclude that $\nu$ is constant. Properties (1.4), (1.5) imply that $\hat{N}(\cdot, s)$ has an inverse $\hat{\nu}(\cdot, s)$ so that (1.11) now implies

$$
\nu=\hat{\nu}\left(N_{0}, s\right)
$$

where $N_{0}$ is the constant value of $N$. Again (1.12) and (1.13) obtain. If $\cos \psi=1$ then $\mathbf{r}=r(s) \mathbf{k}$ with $r^{\prime}(s)=\hat{\nu}\left(N_{0}^{*}, s\right), r(0)=0, r(1)=b . N_{0}^{*}$ is the value of the tension in this case. The relation

$$
\int_{0}^{1} \hat{\nu}\left(N_{0}^{*}, s\right) d s=b
$$


serves to define $N_{0}^{*}$ uniquely. Thus in the case we have the "trivial" solution

$$
\mathbf{r}(s)=\int_{0}^{s} \hat{\nu}\left(N_{0}^{*}, s\right) d s \mathbf{k}
$$

with $N_{0}^{*}$ determined by (1.38). If $|\cos \psi|<1$ we again have (1.15). (1.17) becomes

$$
\theta^{\prime}=\hat{\nu}\left(N_{0}, s\right) \lambda / N_{0} \text {. }
$$

While in place of (1.18) we now have

$$
\int_{0}^{1} \hat{\nu}\left(N_{0}, s\right) \mathbf{e}(s) d s=b \mathbf{k} .
$$

Taking components

$(1.42 \mathrm{a}, \mathrm{b}, \mathrm{c})$

$$
\begin{gathered}
\int_{0}^{1} \hat{\nu}\left(N_{0}, s\right) \cos \theta d s=0, \quad \int_{0}^{1} \hat{\nu}\left(N_{0}, s\right) \sin \theta d s=0, \\
\cos \psi \int_{0}^{1} \hat{\nu}\left(N_{0}, s\right) d s=b .
\end{gathered}
$$

Now using (1.40) we obtain from $(1.42 \mathrm{a}, \mathrm{b})$,

$$
\sin \theta(1)-\sin \theta(0)=0, \quad \cos \theta(1)-\cos \theta(0)=0 .
$$

As before (cf. (1.22)) this leads to

$$
\theta(1)-\theta(0)=2 \pi m, \quad m=1,2,3, \ldots .
$$

But on account of (1.40) this gives

$$
\frac{\lambda}{N_{0}} \int_{0}^{1} \hat{\nu}\left(N_{0}, s\right) d s=2 \pi m, \quad m=1,2,3, \ldots
$$

This is the analog of (1.23). We obtain $\cos \psi$ from (1.42c). We again may use (1.40) to integrate $\mathbf{r}^{\prime}=\nu \mathbf{e}$ to find $\mathbf{r}$. Thus we have

1.45. THEOREM. Suppose $N=\hat{N}(\nu, s)$. Let $\lambda>0$ and $N_{0}$ satisfy $(1.44)$ where $\hat{\nu}(\cdot, s)$ is the inverse function to $\hat{N}(\cdot, s)$. We suppose $\int_{0}^{1} \hat{\nu}\left(N_{0}, s\right) d s>b$. Then problem (1.7), (1.8) has solutions

$(1.46) \mathbf{r}(s)=\left(N_{0} / \lambda\right)\left[\sin \theta(s)-\sin \theta_{0}\right] \sin \psi \mathbf{i}$

$$
\begin{aligned}
+\left(N_{0} / \lambda\right)\left[\cos \theta_{0}-\cos \theta(s)\right] \sin \psi \mathbf{j}+\cos \psi \int_{0}^{s} \hat{\nu}\left(N_{0}, \boldsymbol{\sigma}\right) & d \boldsymbol{\sigma} \mathbf{k}, \\
0 & \leqslant \theta_{0}<2 \pi .
\end{aligned}
$$

Here

$$
\begin{aligned}
& \theta(s)=\theta_{0}+\frac{\lambda}{N_{0}} \int_{0}^{s} \hat{\nu}\left(N_{0}, \sigma\right) d \sigma, \\
& \psi=\arccos b / \int_{0}^{1} \hat{\nu}\left(N_{0}, \sigma\right) d \sigma .
\end{aligned}
$$

In this case if we linearize (1.7) about the trivial solution (1.39) we obtain:

$$
\begin{aligned}
& N_{0}^{*}\left(x_{1}^{\prime} / \hat{\nu}\left(N_{0}^{*}, s\right)\right)^{\prime}+\lambda y_{1}^{\prime}=0, \\
& N_{0}^{*}\left(y_{1}^{\prime} / \hat{\nu}\left(N_{0}^{*}, s\right)\right)^{\prime}-\lambda x_{1}^{\prime}=0,
\end{aligned}
$$




$$
\begin{gathered}
\left(\hat{N}_{\nu}(\hat{\nu}, s) z_{1}^{\prime}\right)^{\prime}=0, \\
x_{1}(0)=y_{1}(0)=z_{1}(0)=x_{1}(1)=y_{1}(1)=z_{1}(1)=0 .
\end{gathered}
$$

Since $\hat{N}_{v}>0,(1.49 \mathrm{c})$ with the boundary conditions $(1.49 \mathrm{~d})$ has only the trivial solution $z_{1} \equiv 0$. We can combine (1.49a) and (1.49b) by writing $\zeta=x_{1}+i y_{1}$. We then obtain

$$
\begin{gathered}
N_{0}^{*}\left(\zeta^{\prime} / \hat{\nu}\right)^{\prime}-\lambda i \zeta^{\prime}=0, \\
\zeta(0)=\zeta(1)=0 .
\end{gathered}
$$

Thus, integrating once

$$
N_{0}^{*}\left(\zeta^{\prime} / \nu\right)-\lambda i \zeta=C .
$$

This has the solution (satisfying $\zeta(0)=0$ )

$$
\zeta(s)=\frac{i C}{\lambda}\left[1-\exp \frac{\lambda i}{N_{0}^{*}} \int_{0}^{s} \hat{\nu}\left(N_{0}^{*}, \sigma\right) d \sigma\right] .
$$

To satisfy the boundary condition at $s=1$ we must then have

$$
\frac{\lambda}{N_{0}^{*}} \int_{0}^{1} \hat{\nu}\left(N_{0}^{*}, \sigma\right) d \sigma=2 \pi m, \quad m=1,2,3, \ldots
$$

Thus we obtain the desired bifurcation relation. Note that (1.50) yields a two-dimensional real eigenspace for $(1.49 \mathrm{a}, \mathrm{b}, \mathrm{c}, \mathrm{d})$ as the constant $C$ in (1.50) can be complex.

2. Linearized stability. The dynamical problem of the motion of the elastic conductor in a magnetic field is governed by the hyperbolic equation

$$
\rho(s) \mathbf{r}_{t t}=\mathbf{n}^{\prime}(s)+\mathbf{f}(s),
$$

with $\mathbf{f}$ given by (1.1) and $\mathbf{n}$ by (1.3) and (1.6). We assume that $\hat{N}$ does not depend explicitly on $s$ and $\rho$ is constant. We will also assume condition (1.30). We will study (2.1) by linearizing about the nontrivial solutions found in $\S 1$. Our results will support the idea that the first branch $(m=1)$ of solutions is in some sense stable while the others are not.

Let $L_{\mathbf{r}}$ be the linearization of the operator on the left-hand side of (1.7). Let the solution (1.25) (with $\theta_{0}=0$ for convenience) be denoted by

$$
\mathbf{r}(s)=x_{0} \mathbf{i}+y_{0} \mathbf{j}+z_{0} \mathbf{k} .
$$

Then $L=L_{\mathbf{r}}$ has the form

$$
L \mathbf{u}=\frac{\hat{N}(\nu)}{\nu} I \mathbf{u}^{\prime \prime}+\left(\frac{\hat{N}_{\nu}(\nu)}{\nu^{2}}-\frac{\hat{N}(\nu)}{\nu^{3}}\right)\left(A \mathbf{u}^{\prime \prime}+B \mathbf{u}^{\prime}\right)+\lambda C u^{\prime} .
$$

Here

$$
A=\left(\begin{array}{ccc}
x_{0}^{\prime} & 0 & 0 \\
0 & y_{0}^{\prime} & 0 \\
0 & 0 & z_{0}^{\prime}
\end{array}\right)\left(\begin{array}{ccc}
x_{0}^{\prime} & y_{0}^{\prime} & z_{0}^{\prime} \\
x_{0}^{\prime} & y_{0}^{\prime} & z_{0}^{\prime} \\
x_{0}^{\prime} & y_{0}^{\prime} & z_{0}^{\prime}
\end{array}\right)
$$




$$
\begin{gathered}
B=\left(\begin{array}{ccc}
2 x_{0}^{\prime \prime} x_{0}^{\prime} & \left(x_{0}^{\prime} y_{0}^{\prime}\right)^{\prime} & x_{0}^{\prime \prime} z_{0}^{\prime} \\
\left(x_{0}^{\prime} y_{0}^{\prime}\right)^{\prime} & 2 y_{0}^{\prime \prime} y_{0}^{\prime} & y_{0}^{\prime \prime} z_{0}^{\prime} \\
z_{0}^{\prime} x_{0}^{\prime \prime} & z_{0}^{\prime} y_{0}^{\prime \prime} & 0
\end{array}\right), \\
C=\left(\begin{array}{ccc}
0 & 1 & 0 \\
-1 & 0 & 0 \\
0 & 0 & 0
\end{array}\right) .
\end{gathered}
$$

From (1.25), (1.23)

$$
\begin{array}{ll}
x_{0}^{\prime}=\nu \sin \psi \cos 2 m \pi s, & x_{0}^{\prime \prime}=-2 m \pi \nu \sin \psi \sin 2 m \pi s, \\
y_{0}^{\prime}=\nu \sin \psi \sin 2 m \pi s, & y_{0}^{\prime \prime}=2 m \pi \nu \sin \psi \cos 2 m \pi s, \\
z_{0}^{\prime}=\nu \cos \psi, & z_{0}^{\prime \prime}=0 .
\end{array}
$$

Let $P$ be the orthogonal matrix

$$
P=\left(\begin{array}{ccc}
\cos 2 \pi m s \sin \psi & -\sin 2 \pi m s & -\cos 2 \pi m s \cos \psi \\
\sin 2 \pi m s \sin \psi & \cos 2 \pi m s & -\sin 2 \pi m s \cos \psi \\
\cos \psi & 0 & \sin \psi
\end{array}\right) .
$$

After a long, but straightforward, computation we find that if

$$
\begin{gathered}
\mathbf{u}=P \mathbf{v}, \quad \mathbf{v}=\left(\begin{array}{c}
x(s) \\
y(s) \\
z(s)
\end{array}\right), \\
P^{t} L P \mathbf{v}=\tilde{A} \mathbf{v}^{\prime \prime}+2 \pi m \tilde{B} \mathbf{v}^{\prime}+4 \pi^{2} m^{2}\left(\hat{N}_{\nu}-\hat{N} / \nu\right) \tilde{C} \mathbf{v}
\end{gathered}
$$

where$$
\tilde{A}=\left(\begin{array}{ccc}
\hat{N}_{\nu} & 0 & 0 \\
0 & \hat{N} / \nu & 0 \\
0 & 0 & \hat{N} / \nu
\end{array}\right)
$$$$
\tilde{B}=\left(\begin{array}{ccc}
0 & -\hat{N}_{\nu} \sin \psi & 0 \\
\hat{N}_{\nu} \sin \psi & 0 & -(\hat{N} / \nu) \cos \psi \\
0 & (\hat{N} / \nu) \cos \psi & 0
\end{array}\right) \text {, }
$$$$
\tilde{C}=\left(\begin{array}{ccc}
0 & 0 & 0 \\
0 & -\sin ^{2} \psi & 0 \\
0 & 0 & 0
\end{array}\right)
$$

Finally if $\langle\cdot, \cdot\rangle$ represents the inner product in $\left[L^{2}(0,1)\right]^{3}$ we have

$$
\begin{aligned}
\langle L \mathbf{u}, \mathbf{u}\rangle & =\langle L P \mathbf{v}, P \mathbf{v}\rangle=\left\langle P^{t} L P \mathbf{v}, \mathbf{v}\right\rangle \\
= & \int_{0}^{1}\left\{-\hat{N}_{\nu}\left(x^{\prime}-2 \pi m y \sin \psi\right)^{2}-\frac{\hat{N}}{\nu}\left(z^{\prime}+2 \pi m y \cos \psi\right)^{2}\right. \\
& \left.\quad+\frac{\hat{N}}{\nu}\left(4 \pi^{2} m^{2} y^{2}-y^{\prime 2}\right)\right\} d s .
\end{aligned}
$$


We are interested in the spectrum of the selfadjoint operator $L$ acting on $\left[H_{2}(0,1)\right]^{3}$ $\cap\left[H_{1}^{0}(0,1)\right]^{3}$. For $m>1,\langle L \mathbf{u}, \mathbf{u}\rangle$ takes on positive values. In fact if

(2.13) $x=m \sin \psi(1-\cos 2 \pi s), \quad y=\sin 2 \pi s, \quad z=m \cos \psi(\cos 2 \pi s-1)$,

for the corresponding $\mathbf{u}=\mathbf{u}_{m}^{*}$,

$$
\left\langle L \mathbf{u}_{m}^{*}, \mathbf{u}_{m}^{*}\right\rangle=(\hat{N} / \nu) \cdot 2 \pi^{2}\left(m^{2}-1\right) .
$$

Thus if $m>1$ and

$$
\mathbf{u}=P \mathbf{v}, \quad \mathbf{v}=\left(\begin{array}{l}
x \\
y \\
z
\end{array}\right)
$$

given by (2.13), then $\langle L \mathbf{u}, \mathbf{u}\rangle>0$. Thus $L$ has a positive eigenvalue. This implies that the branches for $m>1$, are unstable.

We now consider the case $m=1$. For $\mathbf{u}_{1}^{*}$ corresponding to (2.13) with $m=1$, $\left\langle L \mathbf{u}_{1}^{*}, \mathbf{u}_{1}^{*}\right\rangle=0$. But

$$
\mathbf{u}_{1}^{*}=\left(\begin{array}{l}
x_{1} \\
y_{1} \\
z_{1}
\end{array}\right)=P\left(\begin{array}{l}
x \\
y \\
z
\end{array}\right)
$$

is given by

$$
x_{1}=\cos 2 \pi s-1, \quad y_{1}=\sin 2 \pi s, \quad z_{1}=0 .
$$

But by differentiating (1.25) with respect to $\theta_{0}$ and setting $\theta_{0}=0$ we see that the vector whose components are given by (2.15) is tangent to the curve of solutions (1.25). Since $\Phi$ (given by (1.32)) is constant along this curve and formally

$$
\delta^{2} \Phi(\mathbf{u})=-\langle L \mathbf{u}, \mathbf{u}\rangle,
$$

this result is to be expected.

We now show that for $\mathbf{u}$ not in the subspace spanned by $\mathbf{u}_{1}^{*},\langle L \mathbf{u}, \mathbf{u}\rangle<0$. We consider (2.13) for $m=1$ with $x, y, z \in \stackrel{\circ}{H}_{1}(0,1)$. Then $x=\sum_{n=1}^{\infty} x_{n} \sin n \pi s, x^{\prime}=$ $\pi \sum_{n=1}^{\infty} n x_{n} \cos n \pi s$, etc. Now for a fixed $y$ we choose $x$ so that $\int_{0}^{1}\left(x^{\prime}-2 \pi y \sin \psi\right)^{2} d s$ is as small as possible, i.e., we minimize the square of the distance from $2 \pi y \sin \psi$ to the subspace of $L_{2}(0,1)$ spanned by $\{\cos n \pi s\}_{n=1}^{\infty}$. Since $\{\cos n \pi s\}_{n=0}^{\infty}$ is an orthogonal basis for $L_{2}(0,1)$ this minimum will, by standard Hilbert space theory, be $\left(\int_{0}^{1} 2 \pi y \sin \psi d s\right)^{2}=4 \pi^{2} \sin ^{2} \psi\left(\int_{0}^{1} y d s\right)^{2}$, i.e.,

$$
\int_{0}^{1}\left(x^{\prime}-2 \pi y \sin \psi\right)^{2} d s \geqslant 4 \pi^{2} \sin ^{2} \psi\left(\int_{0}^{1} y d s\right)^{2} .
$$

In the same way

$$
\int_{0}^{1}\left(z^{\prime}+2 \pi y \cos \psi\right)^{2} d s \geqslant 4 \pi^{2} \cos ^{2} \psi\left(\int_{0}^{1} y d s\right)^{2}
$$

Hence for $m=1$,

$$
\begin{aligned}
\langle L \mathbf{u}, \mathbf{u}\rangle \leqslant & -4 \pi^{2}\left(\hat{N}_{\nu} \sin ^{2} \psi+\frac{\hat{N}}{\nu} \cos ^{2} \psi\right)\left(\int_{0}^{1} y d s\right)^{2} \\
& +\frac{\hat{N}}{\nu} \int_{0}^{1}\left(4 \pi y^{2}-y^{\prime 2}\right) d s .
\end{aligned}
$$


Now by (1.30), $\hat{N}_{\nu}>\hat{N} / \nu$, thus

$$
\langle L \mathbf{u}, \mathbf{u}\rangle \leqslant \frac{\hat{N}}{\nu}\left\{-4 \pi^{2}\left(\int_{0}^{1} y d s\right)^{2}+\int_{0}^{1}\left(4 \pi^{2} y^{2}-y^{\prime 2}\right) d s\right\} .
$$

Thus the proof will be complete if we can show

$$
4 \pi^{2} \int_{0}^{1} y^{2} d s \leqslant 4 \pi^{2}\left(\int_{0}^{1} y d s\right)^{2}+\int_{0}^{1} y^{\prime 2} d s, \quad y \in \stackrel{\circ}{H}_{1}(0,1),
$$

and examine the cases in which equality holds (e.g., $y=\sin 2 \pi s$ which we have already considered).

The following proof of (2.19) was suggested by J. Ullman.

Write $y=a_{0}+\sum_{n=1}^{\infty}\left(a_{n} \cos 2 \pi n s+b_{n} \sin 2 \pi n s\right)$. Then (2.19) is equivalent to

$$
4 \pi^{2}\left\{a_{0}^{2}+\frac{1}{2} \sum_{n=1}^{\infty}\left(a_{n}^{2}+b_{n}^{2}\right)\right\} \leqslant 4 \pi^{2} a_{0}^{2}+2 \pi^{2} \sum_{n=1}^{\infty}\left(n^{2} a_{n}^{2}+n^{2} b_{n}^{2}\right),
$$

which is clearly true. Equality holds if and only if $a_{n}=b_{n}=0, n \geqslant 2$. (Note that (2.20) is true for $y \in H_{1}(0,1)$.) For functions in $\dot{H}_{1}$ equality holds in (2.20) only for functions of the form $y=c_{1} \sin 2 \pi s+c_{2}(1-\cos 2 \pi s)$. But if $c_{2} \neq 0, \int_{0}^{1} y(s) d s \neq 0$. In this case inequality (2.18) is strict. When $y=\sin \pi s$ in order to have $\langle L \mathbf{u}, \mathbf{u}\rangle=0$, we see that

$$
\left(\begin{array}{l}
x \\
y \\
z
\end{array}\right)
$$

must have the form (2.13) with $m=1$ and, hence, $\mathbf{u}=\mathbf{u}_{1}^{*}$. So we have

2.21. Theorem. Assume (1.30) holds. $\mathbf{r}_{0}$ is given by (1.25), (1.23) with $m=1$, $\theta_{0}=0$. Let $L$ be the linearization of the operator $\mathbf{r} \rightarrow(\hat{N}(\nu) \mathbf{e})^{\prime}+\lambda \nu \mathbf{e} \times \mathbf{k}$ at $\mathbf{r}_{0}$. Then for $\mathbf{u} \in\left[\stackrel{H}{1}_{1}(0,1)\right]^{3} \cap\left[H_{2}(0,1)\right]^{3},(L \mathbf{u}, \mathbf{u}) \leqslant 0$ with equality if and only if $\mathbf{u}=c \mathbf{u}_{1}^{*}$ where $\mathbf{u}_{1}^{*}$ has components given by (2.15). The vector $\mathbf{u}_{1}^{*}$ is in the direction of the tangent to the solution curve (1.25).

2.22. Corollary. Theorem 2.21 remains true if we replace $\theta_{0}=0$ by an arbitrary $\theta_{0}, 0<\theta_{0}<2 \pi$.

Proof. Perform a rotation of coordinates in the $(x, y)$ plane.

The preceding results lead us to conjecture that the set $S$ of solutions (1.23), (1.25) with $m=1$ forms a stable invariant manifold for (2.1) in the sense that if we consider a solution of (2.1) with $\mathbf{r}(\cdot, 0)$ close to $S$ and $\mathbf{r}_{t}(\cdot, 0)$ small (in some suitable sense), the solution will exist for all $t>0$ and remain close to $S$. For classical solutions of (2.1) with constant $\rho$ the quantity

$$
E=\frac{\rho}{2} \int_{0}^{1}\left|\mathbf{r}_{t}\right|^{2} d s+\Phi
$$


is conserved. However $\Phi$ is not necessarily continuous in the $\left[H_{1}\right]^{3}$ norm so the relevance of (2.23) is not clear. We also believe that if we consider models of the motion of the wire with a physically reasonable dynamic dissipation mechanism, solutions will tend to some point of $S$ as $t \rightarrow \infty$.

\section{BIBLIOGRAPHY}

1. F. C. Moon, Problems in magneto-solid mechanics, Mechanics Today, Vol 4 (Nemat-Nasser, editor), Pergamon Press, Oxford, 1978.

Department of Mathematics, University of Michigan, Ann Arbor, Michigan 48109

Department of Mathematics, University of Maryland, College Park, Maryland 20742 